\title{
Recycling Of Ceramic Refractory Materials
}

\author{
H. Baloyi, Bm Thethwayo And Antoine F. Mulaba - Bafubiandi
}

\begin{abstract}
Refractories are structural materials that can withstand high temperatures and corrosive environments. These materials are used in lining reactors used in producing metals, glass etc. In our laboratory we use ceramic furnace tubes made of alumina, silica or magnesia. These tubes are costly, yet they are prone to cracking and wear due to chemical reactions and thermal shock, suppliers do not accept these tubes after use hence a way of recycling these materials has to be established so the tubes can be reused since they affect the environment negatively when dumped in landfills. Refractory materials disposal has become a major problem for metal producers. As chromium (Cr2O3) can be oxidized to $\mathrm{Cr}$ (CrO4-2, refractory materials containing chromium have more problems as harmful hexavalent chromium ions may be formed under process circumstances). Spent furnace tubes (alumina) were collected from pyrometallurgy labs. They were either broken due to thermal shock or chemical reactions. Two types of tubes tested were alumina and alumina-silicate tubes. Alumina tubes were further classified to high and moderate wear. These tubes were hand sorted based on appearance (color and extent of wear). The tubes were crushed, milled, screened and purified using a shaking table and a magnetic separator. Alumina content was upgraded from $91,2 \%$ to $94,8 \%$, whereas iron content was decreased from $3.7 \%$ to $2.2 \%$ using a shaking table. For alumina-silicate tube, alumina was upgraded from $54,1 \%$ to $58,0 \%$. Iron content did not change much as it has increased from $2.4 \%$ to $2.5 \%$, using a shaking table. Purified powders were formed into crucibles using bentonite as a binder.
\end{abstract}

Keywords - Alumina tubes, alumina silicates, recycle, refractory materials.

\section{INTRODUCTION}

Refractories are strong materials which can resist elevated temperatures. These materials should maintain their mechanical functions under all conditions, even in contact with corrosive liquids or gases. Refractories are used as lining for vessels used in metal manufacturing, glass, cement and ceramics for elevated temperature procedures (Horckmans, Nielsen, Dierckx, \& Ducastel, 2019).

\section{Why SHOULD THEY BE RECYCLED?}

Refractory materials that contain copper, chrome, bauxite and magnesite cause environmental hazards. Refractories with chromium are considered more harmful to human beings and the environment due to its toxicity. When such refractories are exposed to the environment, chromium $\left(\mathrm{Cr}_{2} \mathrm{O}_{3}\right)$ can be oxidized to $\mathrm{Cr}\left(\mathrm{CrO}_{4}^{-2}\right)$. When chromium (VI) is exposed to the environment, it can cause cancer. Wastes materials that contain chromium cannot be discarded because they are considered high risk materials (Simon, Adamczyk, \& Kley, 2003).

H. Baloyi, Bm Thethwayo And Antoine F. Mulaba - Bafubiandi, University of Johannesburg, South Africa.
Furnace refractory tubes are exposed to high temperatures, corrosive and thermal cycling. During operation, tubes can undergo wear due to 3 methods such as thermal shock, chemical shock. Refractory wear can be classified into three wears, namely: physical, chemical (dissolution) and mechanical wear (thermal shock). Physical wear occurs when there is a physical penetration of a fluid without reactions. Typical alumina tubes are very dense, this makes the tubes' physical penetration not to be observed normally.

Chemical wear is a dissolution where refractory materials chemically react with the fluid forming new products. Chemical wear is seen by discoloration, deformation, etc., as seen in fig 2. The tubes become very dark inside, and some deformation and discoloration cracks can be observed. Thermal shock occurs when there is high temperature gradient in the tube, e.g. a tube at $1000^{\circ} \mathrm{C}$ is torched by a crucible at 40 ${ }^{\circ} \mathrm{C}$. normally, there is no discoloration for thermal shock, the tube just cracks rather (Curzio, 2000). An example of a cracked tube is shown in Fig 1.

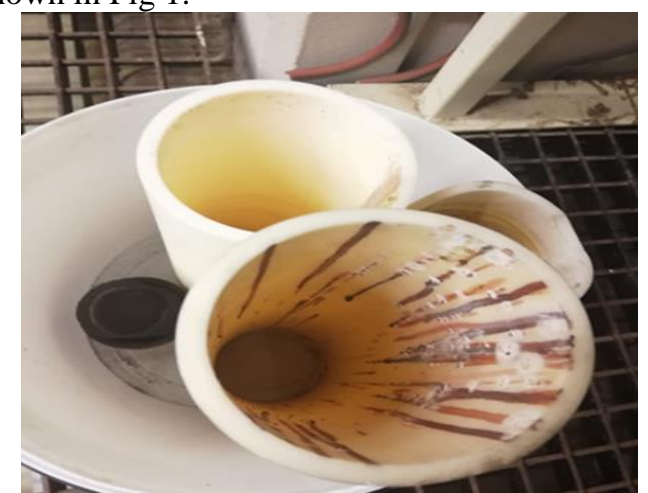

Fig 1: Alumina tubes, cracked due to thermal shock

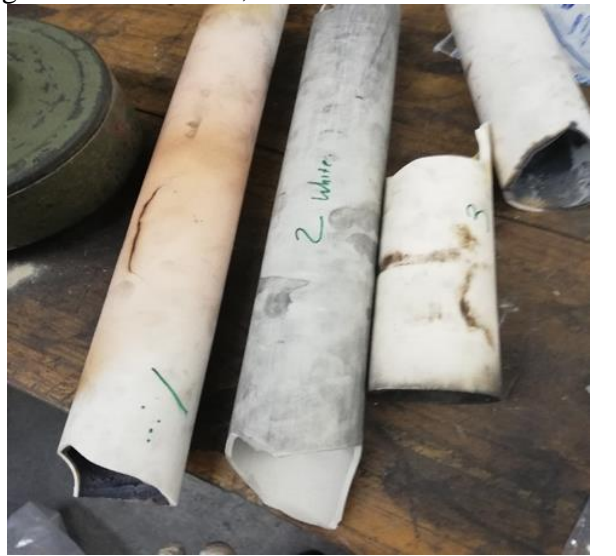

Fig 2: Alumina and alumina silicate tubes that suffered chemical attack and thermal shock

It is expensive to acquire high quality tubes and since suppliers do not accept spent refractory tubes, the tubes are discarded after use. For economic reasons, it is vital to explore feasible methods of recycling spent refractory tubes. The 
recycled tube materials can be used as a monolithic for unshaped refractory mixes, etc.

\section{CHARACTERIZATION}

Tubes were collected from different pyrometallurgical labs. Tubes were categorized based on color and degree of wear, denoted as tubes 1, 2 and 3. Tube 1 was moderately worn with only typical impurities (refer to Fig 1). Tube 2 was yellowish in color and had undergone chemical wear based on dissolution and cracks (refer to Fig 2). Tube 3 seemed greyish in color and had minor chemical wear but possible thermal shock (refer to Fig 2). All these tubes were treated with crushing, milling and shaking table to compare.

Before the tubes were recycled, they were firstly characterized to obtain their compositions. Based on their compositions obtained from X-ray fluorescence (XRF), it was then decided on which methods to be used to recycle the spent tubes. The analyses obtained from XRF are shown by the table 1 below:

$$
\text { TABLE I: SUMMARY OF XRF ANALYSIS }
$$

\begin{tabular}{|c|c|c|c|}
\hline Important Phases & Tube 1 & Tube 2 & Tube 3 \\
\hline $\mathbf{A I}_{2} \mathbf{O}_{3}$ & $\mathbf{9 1 , 2 3 3 2}$ & $\mathbf{9 2 , 4 6 7 1}$ & $\mathbf{5 4 , 1 3 5 5}$ \\
\hline $\mathrm{Fe}_{2} \mathbf{O}_{3}$ & $\mathbf{3 , 6 5 4 2}$ & $\mathbf{2 , 2 3 9}$ & $\mathbf{2 , 3 8 7 6}$ \\
\hline $\mathrm{SiO}_{2}$ & $\mathbf{2 , 1 5 7 9}$ & $\mathbf{1 , 3 3 5 4}$ & $\mathbf{3 9 , 7 2 3 6}$ \\
\hline $\mathrm{MnO}$ & - & $\mathbf{2 , 7 6 9 1}$ & - \\
\hline $\mathrm{TiO}_{2}$ & - & - & $\mathbf{1 , 1 1 4 5}$ \\
\hline $\mathbf{K}_{2} \mathbf{O}$ & - & - & $\mathbf{1 , 4 5 1 4}$ \\
\hline Others & $\mathbf{2 , 9 5 4 7}$ & $\mathbf{1 , 1 8 9 4}$ & $\mathbf{1 , 1 8 7 4}$ \\
\hline
\end{tabular}

From the analyses obtained from XRF, they show that tubes 1 and 2 seemed to be alumina-based tubes. The highest impurity in these tubes was found to be $\mathrm{Fe}_{2} \mathrm{O}_{3}$. $\mathrm{SiO} 2$ was another impurity in both tubes. Tube 2 also had $\mathrm{MnO}$ as another impurity. Tube 3 is different from tubes 1 and 2. Tube 3 is essentially made of alumina and silica. It also contains impurities such $\mathrm{Fe}_{2} \mathrm{O}_{3}$, titanium dioxide $\left(\mathrm{TiO}_{2}\right)$ and potassium oxide $\left(\mathrm{K}_{2} \mathrm{O}\right)$. Other phases such as $\mathrm{Cu}, \mathrm{Cr}$, Ba, etc, have no huge impacts on the tubes. Some of the elements have been purposefully added to enhance the properties of the tubes. The principal aim was to upgrade the alumina content of tubes 1 and 2 , reduce $\mathrm{Fe}_{2} \mathrm{O}_{3}$ and $\mathrm{SiO} 2$. In tube 3, we aimed to increase $\mathrm{Al}$ and $\mathrm{Si}$ content while decreasing the impurities.

\section{RESUlTS AND DiscUSSION}

Tubes 1,2 and 3 were received as seen in fig. 1 and 2. The tubes were initially crushed by jaw and roll crusher to reduce the size before milling to less than $1000 \mu \mathrm{m}(1 \mathrm{~mm})$. To determine the milling curves/grindability, tubes were milled for $10,20,30$ and 60 minutes. The resulting graphs are shown in fig. 3,4 and 5 .

\section{Milling CURVES}
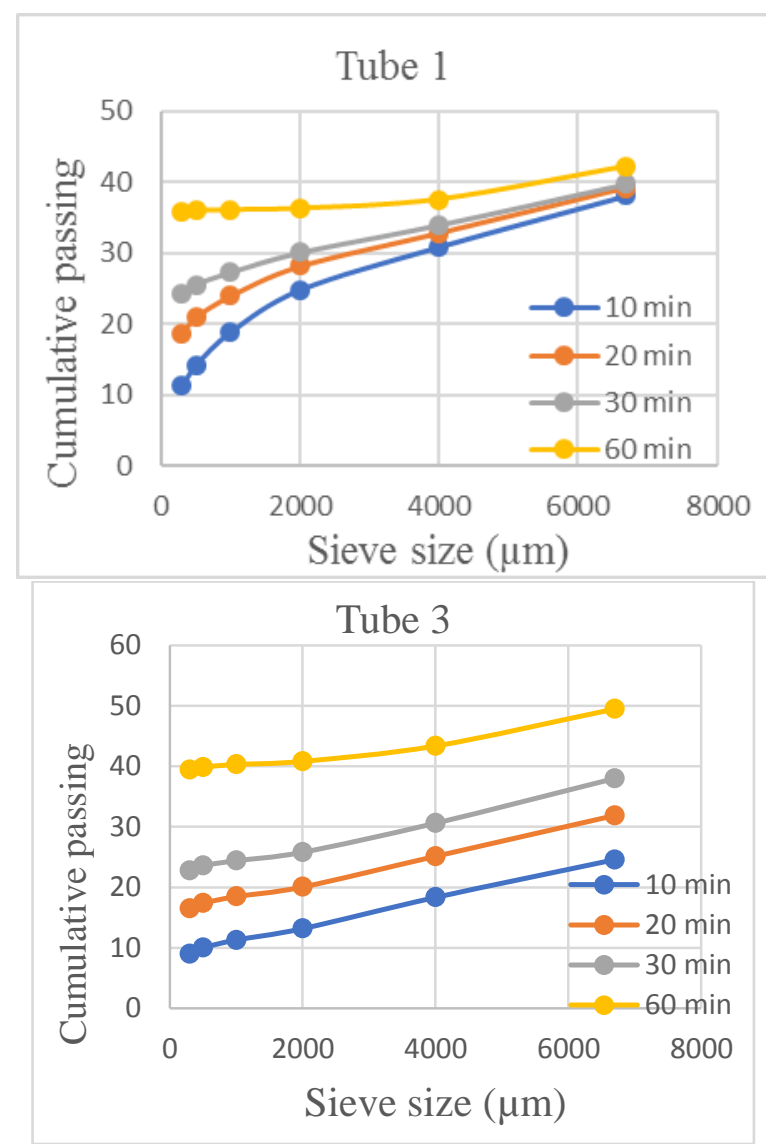

Fig 3: The milling curve for tube 1 and tube 3

From the graph above for tube 1, the results show that as the milling time increases, the cumulative passing also increases. During time intervals 10, 20 and 30 minutes, at a sieve size of $1000 \mu \mathrm{m}$, the cumulative passing increases from approximately 19 to approximately 27 . This is because alumina tubes have a high hardness, this makes them not to be easily milled. After 60 minutes, the cumulative passing increases greatly to approximately 35 as more time is spent.

Tube 2 is also made mainly of alumina, which is a very hard material. From the results obtained, cumulative passing increases with an increasing milling time. This is the same as in tube 1. Between milling times 10, 20 and 30 mins, at a sieve size of $1000 \mu \mathrm{m}$, the cumulative passing increases from approximately 7 to approximately 17 . Tube 2 was less grindable with only approximately $15 \%$ cumulative passing $1000 \mu \mathrm{m}$. it was then removed from the results because it is almost the same as tube 1 .

From the graph above, the cumulative passing for tube 3 is slightly different from tubes 1 and 2 . Between milling times 10 , 20 and 30 mins, the cumulative passing increases from approximately 11 to approximately 26 at a sieve size of 1000 $\mu \mathrm{m}$. Tube 3 is mainly made of alumina and silica, it is not as hard as tubes 1 and 2, which makes the milling of this tube a bit simpler than the two tubes. Tube 3 reached $40 \%$ cumulative passing after 60 mins. The hardness of $\mathrm{Al}-\mathrm{Si}$ is lower than that of alumina (9 Mohs hardness). Based on the grindability of tubes 1 and 2, it seems that exposure to operational conditions may affect the hardness of the refractory material. 


\section{SHAKING TABLE}

Tubes 1, 2 and 3 were treated in a shaking table to upgrade the refractory material. XRF was used to analyze the products. The analyses are in table 2.

TABLE II: XRF ANALYSIS FOR SHAKING TABLE

\begin{tabular}{|c|c|c|c|c|c|r}
\hline \multirow{2}{*}{ Important Phases } & \multicolumn{3}{|c}{ Tails } & \multicolumn{3}{c}{ Concentrates } \\
\cline { 2 - 7 } & Tube 1 & Tube 2 & Tube 3 & Tube 1 & Tube 2 & Tube \\
\hline $\mathbf{A l}_{2} \mathbf{O}_{3}$ & $\mathbf{9 5 , 1}$ & $\mathbf{9 4 , 8}$ & $\mathbf{5 8 , 0}$ & $\mathbf{8 4 , 6}$ & $\mathbf{9 2 , 8}$ & $\mathbf{5 2 , 5}$ \\
\hline $\mathrm{Fe}_{2} \mathbf{O}_{3}$ & $\mathbf{2 , 0}$ & $\mathbf{2 , 2}$ & $\mathbf{2 , 5}$ & $\mathbf{6 , 4}$ & $\mathbf{2 , 4}$ & $\mathbf{5 , 4}$ \\
\hline $\mathrm{SiO}_{2}$ & $\mathbf{1 , 5}$ & $\mathbf{1 , 4}$ & $\mathbf{3 5 , 9}$ & $\mathbf{4 , 4}$ & $\mathbf{3 , 0}$ & $\mathbf{3 5 , 2}$ \\
\hline $\mathrm{MnO}$ & - & - & - & - & - & - \\
\hline $\mathrm{TiO}_{2}$ & - & - & - & - & - & $\mathbf{1 , 8}$ \\
\hline $\mathrm{K}_{2} \mathbf{O}$ & - & - & $\mathbf{1 , 2}$ & - & - & $\mathbf{2 , 2}$ \\
\hline Others & $\mathbf{1 , 3}$ & $\mathbf{1 , 5}$ & $\mathbf{2 , 4}$ & $\mathbf{2 , 8}$ & $\mathbf{1 , 8}$ & $\mathbf{2 , 9}$ \\
\hline
\end{tabular}

From the results obtained after shaking table, alumina in tube 1 was upgraded from $91,2 \%$ \& to $95,1 \%$, while $\mathrm{Fe} 2 \mathrm{O} 3$ has been downgraded from $3,7 \%$ to $2,0 \%$. SiO2 was as well decreased from $2,2 \%$ to $1,5 \%$. The alumina in tube 2 was also upgraded from $92,5 \%$ to $94,8 \%$. $\mathrm{Fe} 2 \mathrm{O} 3$ and $\mathrm{SiO} 2$ did not change much. For tube 3, alumina was upgraded from $54,1 \%$ to $58,0 \%$. $\mathrm{Fe} 2 \mathrm{O} 3$ did not show much of a difference. $\mathrm{SiO} 2$ was downgraded from $39,7 \%$ to $35,9 \%$. Some other elements that did not have much impact were added together.

\section{Procedure For Producing A Crucible}

Shaking table tails were mixed with bentonite to make a crucible. $200 \mathrm{ml}$ of water was added to a mixture. The mixture was then shaped and dried for 24 hours after which it was dried in the oven at $105^{\circ} \mathrm{C}$ for two days. The crucible was further baked at $700^{\circ} \mathrm{C}$ for four hours. The image of the typical crucible to be produced is shown in fig. 6 .
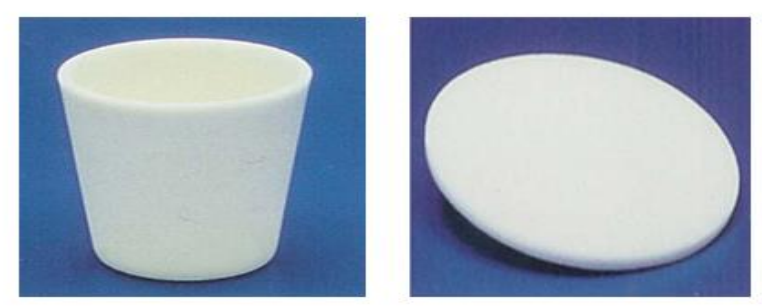

Source:

https://www.ceramicmanufacturing.net/alumina-crucibles/ Fig: Typical crucibles to be produced.

\section{CONCLUSION}

It is not advisable to discard refractory tubes, especially alumina tubes, because of their hazardousness to the environment, and that they cause cancer to human beings. Alumina tubes can be recycled by producing a crucible from a monolithic powder. Shaking table can be used to separate impurities from the tubes.

\section{REFERENCES}

[1] Curzio, E. L. (2000). Properties of CVI-SiC Matrix composites. Comprehensive Composite materials, 4, 533-577. https://doi.org/10.1016/B0-08-042993-9/00112-1

[2] Horckmans, L., Nielsen, P., Dierckx, P., \& Ducastel, A. (2019). Recycling of refractory bricks used in basic steelmaking: A review. Resources, Conservation and Recycling, 140, 297-304.

https://doi.org/10.1016/j.resconrec.2018.09.025
[3] Simon , f. G., Adamczyk, B., \& Kley, G. (2003). Refractory Materials from Waste. Materials Transactions, 44(7), 1251-1254. https://doi.org/10.2320/matertrans.44.1251 